\title{
Sol-Gel Holographic Recording Materials ${ }^{1}$
}

\author{
P. Cheben ${ }^{a}$, M. L. Calvo ${ }^{b}$, F. del Monte ${ }^{c}$, O. Martínez-Matos ${ }^{b}$, and J. A. Rodrigo ${ }^{b}$ \\ ${ }^{a}$ Institute for Microstructural Sciences, National Research Council, Ottawa ON Canada \\ ${ }^{b}$ Departamento de Optica, Facultad de Ciencias Fisicas, Universidad Complutense de Madrid, Madrid, Spain \\ ${ }^{c}$ Instituto de Ciencia de los Materiales, Consejo Superior de Investigaciones Cientificas, Madrid, Spain \\ e-mail: pavel.cheben@nrc.ca \\ Received December 28, 2006
}

\begin{abstract}
The advances in the sol-gel holographic recording materials, namely in the photopolymerizable compositions and photorefractive glasses, are reviewed. A new sol-gel holographic material is also discussed that exploits diffusion of a high-refractive-index MA:Zr complex, with the refractive index modulation of $n \sim$ $10^{-2}$ and negligible scattering.

PACS numbers: 42.40.-i

DOI: $10.1134 / \mathrm{S} 0030400 \mathrm{X} 0712003 \mathrm{X}$
\end{abstract}

\section{INTRODUCTION}

Silica gels are commonly found in nature in the form of opals and agates. The preparation of a synthetic silica gel was first reported by Ebelmen more than 150 years ago [1], laying down the foundations of the sol-gel technique. The fundamental motivation for using sol-gel materials is to replace the high-temperature glass and ceramic fabrication techniques by a process that can take place at lower temperatures, even at room temperature. By avoiding elevated temperatures, it is possible to incorporate organic molecules with low thermal stability into inorganic matrices, resulting in hybrid organic-inorganic materials. Combining the properties of organic and inorganic components in a composite material opens new opportunities in development of innovative materials, including holographic recording media.

\section{Early Experiments with Volume Grating Recording in Sol-Gel Glass}

In 1996, the first organically modified sol-gel material capable of recording volume holograms was demonstrated [2]. This material resulted from the effort to overcome the problems with limited maximum thickness of commercial holographic photopolymers as well as with material shrinkage upon polymerization typical for acrylic-based materials. The basic idea here is to disperse organic photopolymerizable species in an inorganic host matrix rather than in an organic binder such as is typically used for this purpose. The inorganic host matrix can significantly improve physical properties of the holographic recording material, such as its rigidity, environmental stability, dimensional changes

\footnotetext{
${ }^{1}$ The text was submitted by the authors in English.
}

upon holographic exposure, maximum achievable thickness, and the ability to accept an optical-grade polish. The support matrix of this organic-inorganic material, in contrast to the porous glass holographic materials $[3,4]$, was formed by in situ polymerization (sol-gel reaction) of liquid silica precursors in presence of dissolved photoinitiating and photopolymerizable species. The material was prepared in form of a few millimeters thick monoliths, and volume gratings with diffraction efficiency of more than $90 \%$ were holographicaly recorded in it.

Following this strategy, a sol-gel glass was developed with refractive index modulation of $\Delta n \sim 0.004$ and a diffraction efficiency of $98 \%$ for an exposure of $230 \mathrm{~mJ} / \mathrm{cm}^{2}$ at $514.5 \mathrm{~nm}$ wavelength [5]. The material consists of a glassy host containing an ethylenic unsaturated monomer ethylene glycol phenyl ether acrylate and a free radical generating titanocene photoinititiator bis( $\mu^{5}-2,4$-cyclopentadien-1-yl)-bis-[2,6-difluoro-3-1Hpyrrol-1-yl)phenyl]titanium, and was fabricated both as thick films and monoliths. The results obtained with this material implied that sol-gel materials are an important candidate for holographic data storage, and various new sol-gel photopolymerizable compositions have recently emerged [6-8].

\section{Photorefractive Sol-Gel Glass}

Photorefractive gratings with refractive index modulation of 0.002 and a two-beam coupling gain of $444 \mathrm{~cm}^{-1}$ were also demonstrated in an organically modified permanently poled sol-gel glass [9]. The azodye 2,5-dimethyl-4-(2-hydroxyethoxy)-4'-nitroazobenzene (DMHNAB) was used as a nonlinear optical chromophore. The chromophore molecules were covalently bonded to the silica glass backbone in order to achieve 
(a)

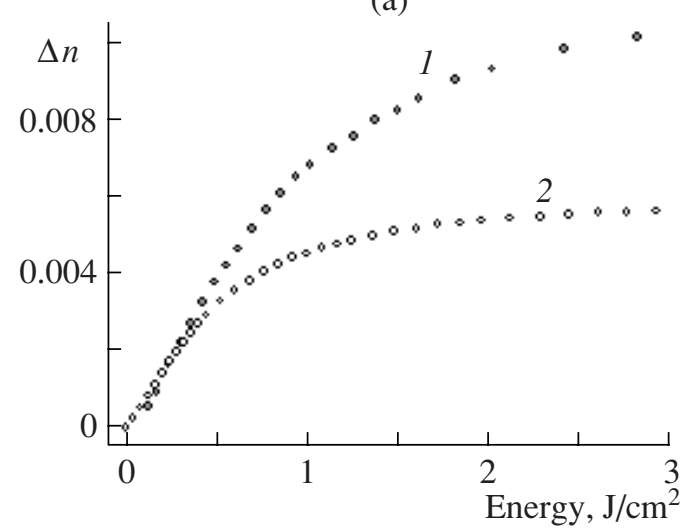

(b)

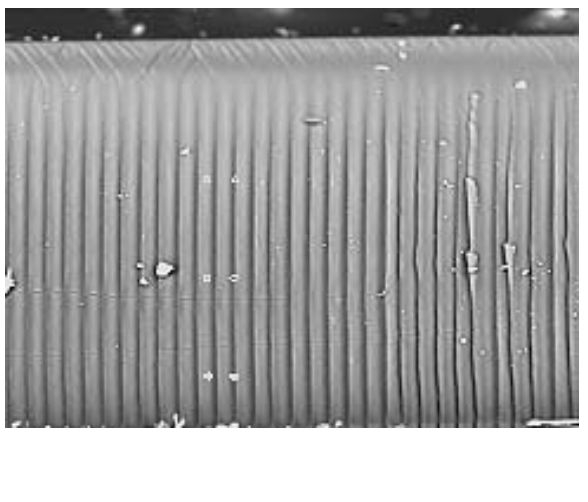

Fig. 1. Refractive index modulation evolution with exposure for the samples with (1) and (2) without the high-index species (a). Samples thickness is $35 \mu \mathrm{m}$. Scanning electron microscope image of a volume holographic grating of a thickness $250 \mu \mathrm{m}$ and a spatial frequency 100 lines/mm recorded in a sample with MA:Zr (b).

the high dye concentration required for efficient nonlinear optical properties, while avoiding the dye crystallization that is typically observed in guest-host photorefractive polymers. 2,4,7-trinitro-9-fluorenone (TNF) was used as photosensitizer and $N$-ethylcarbazole (ECZ) as the charge-transporting agent, both being present as guests in the glass, i.e., without being covalently attached to the matrix. Excellent resistance against chromophore crystallization is achieved by covalently bonding the chromophore. High stability of the electric-field-induced chromophore alignment is due to a gradual heat-induced densification of the gel with initially low glass transition temperature $\left(T_{g}\right)$ during the electric field poling, eventually yielding a high$T_{g}$ hard glassy film. This densification process is essential for slowing down diffusive randomization of the

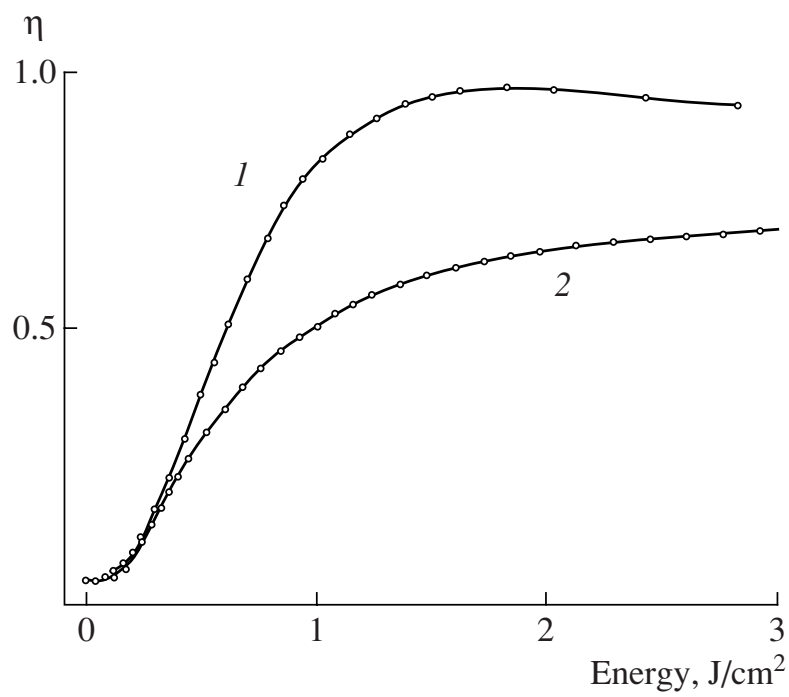

Fig. 2. Diffraction efficiency evolution with exposure for the samples with (1) and without (2) the high-index species. chromophore alignment and for improving the mechanical, electrical, and thermal properties of the glass.

\section{Holographic Sol-Gel Glass Incorporating High Refractive Index Species}

We have recently developed a sol-gel holographic material [10] incorporating in a host similar to our previously reported matrix [5] a high-refractive-index MA:Zr complex (zirconium isopropoxide chelated with metha-acrylic acid), with a two-fold increase in the refractive index modulation compared to our original composition. The improvement resides in the ability of the high-index MA:Zr complex to diffuse upon inhomogeneous illumination. Upon photoinduced polymerization of the metha-acrylic acid, the concentration gradient driven diffusion of the MA: $\mathrm{Zr}$ complex takes place from the dark to the light regions of the interference pattern. As it is shown in Fig. 1, by incorporating in the host the high-index MA:Zr species, the refractive index modulation of the material is increased to $\Delta n \sim$ $10^{-2}$ compared to $\Delta n \sim 5.6 \times 10^{-3}$ in the sample without the high-index species. Furthermore, compared to the photopolymers with dispersed high-index $\left(\mathrm{TiO}_{2}\right)$ nanoparticles $[11,12]$, the scattering is markedly reduced as a consequence of the molecular (rather than nanoparticle) nature of MA:Zr.

The achievement of index modulation of $10^{-2}$ [5] (see Fig. 1, curve for material with MA:Zr) is remarkable and this value is among the best obtained in a holographic photopolymerizable composition of similar thickness. At the same time, photosensitivity is similar to $P O E A$ based photopolymerizable glass [5] and high diffraction efficiencies close to theoretical $100 \%$ limit can be obtained even for relatively thin samples with a Klein-Cook parameter $Q \sim 20$, see Fig. 2. The $Q$ value confirms the volume (Bragg) nature of these gratings, with insignificant contribution of surface modulation, 


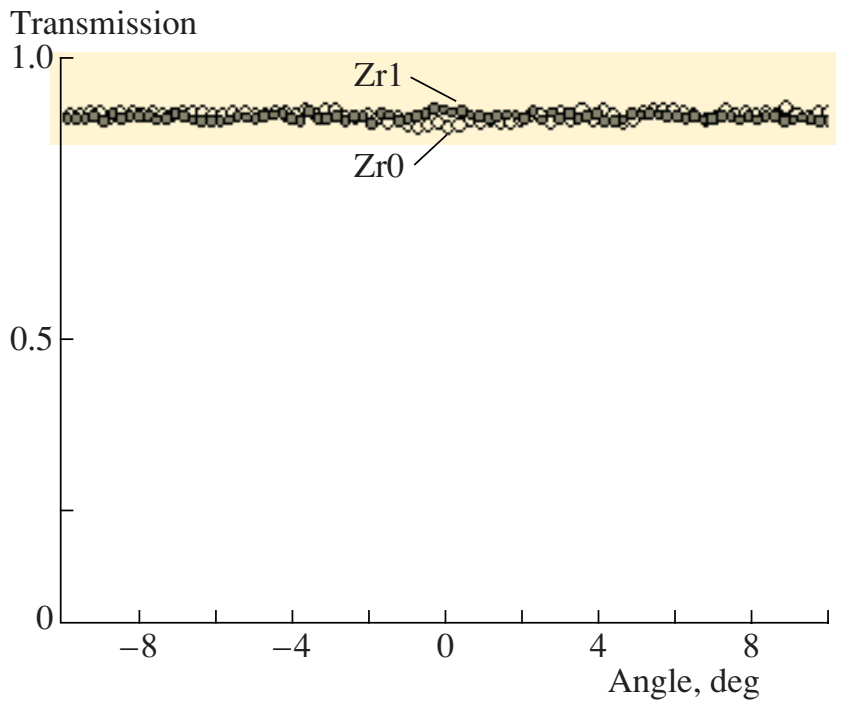

Fig. 3. Noise grating angular selectivity measurement for samples $\mathrm{Zr} 0$ and $\mathrm{Zr} 1$. The samples were exposed and readout by a coherent $s$-polarized single beam of wavelength $532 \mathrm{~nm}$ and intensity $5 \mathrm{~mW} / \mathrm{cm}^{2}$. Exposure is $15 \mathrm{~J} / \mathrm{cm}^{2}$.

as it is also confirmed by the scanning force microscopy (SFM) image shown in Fig. $1 \mathrm{~b}$.

Besides holographic performance, incorporation of high refractive index species in molecular form is crucial for the reduction of coherent and incoherent scattering noise, which is one of the fundamental challenges in holographic data storage applications. Scattering originates in imperfections in the surface and volume material, the latter of which are of particular concern in materials with large thicknesses. Scattered light interfering with incident beams forms noise holograms, thus reducing the dynamic range and deteriorating crosstalk in multiplexed holograms.

To study scattering in our material, we performed the two independent experiments. The first one is the same as the one used by Sanchez et al. [12] in a recent paper on holographic materials where the incorporation of $\mathrm{TiO}_{2}$ nanoparticles provides $\Delta n$ on the order of $10^{-2}$. The scattering coefficient $\alpha$ found for $\mathrm{ZrO}$ (without MA : Zr) and Zr1 (with MA : Zr) samples are $2 \times 10^{-3}$ and $1.2 \times 10^{-3} \mu \mathrm{m}^{-1}$, respectively. Note that for a material of $15 \mu \mathrm{m}$ thickness, these values would correspond to $3 \%$ ( $\mathrm{Zr} 0$ ) and $\sim 1.8 \%$ (Zr1) of scattered light, which demonstrate about 8 -fold noise reduction compared to that reported for $\mathrm{TiO}_{2}$ nanoparticles based holographic material. To further corroborate the low level of scattering, we perform a second experiment previously used for characterization on Aprilis ULSH photopolymer [13]. There is virtually no transmittance degradation in $\mathrm{Zr} 1$ sample, indicating that the scattering is very weak regardless on the incorporation of MA:Zr. This very weak character of the noise grating was also confirmed by the noise grating angular selectivity measurement shown in Fig. 3. In the presence of noise grating, a dip in diffraction efficiency is expected near the Bragg resonance, which is virtually absent in our samples. Experiments performed with $p$-polarized writing beams show identical results.

\section{CONCLUSIONS}

At present, sol-gel materials are among the best performing holographic recording media. They offer a large refractive index modulation, high sensitivity and dimensional stability, and low scattering. Research is being continued by the authors to extend the present studies, namely to further enhance the holographic properties, as well as to explore the applications, of this material.

\section{ACKNOWLEDGMENTS}

Partial financial support from the Spanish Ministry of Education and Science, under grant TEC2005-2180 is acknowledged.

\section{REFERENCES}

1. J. J. Ebelmen, C. R. Acad. Sci. 19, 398 (1844).

2. P. Cheben, T. Belenguer, A. Núñez, et al., Opt. Lett. 21, 1857 (1996).

3. E. A. Chandros, W. J. Tomlinson, and G. D. Aumiller, Appl. Opt. 17, 566 (1978).

4. V. I. Sukhanov, M. V. Kazankova, A. M. Kursakova, and O. V. Andreeva, Opt. Spectrosc. 65, 282 (1988).

5. P. Cheben and M. L. Calvo, Appl. Phys. Lett. 78, 1490 (2001). Press releases on this sol-gel material: Nature 422, 556 (2003); Nature Science Update: http:// www.nature.com/nsu/010315/010315-7.html; New York Times: http://www.nytimes.com/2001/07/12/technology/ circuits/12NEXT.html; Physics Today: http://www. physicstoday.org/pt/vol-54/iss-5/p9.html; Chemical and Engineering News: http://pubs.acs.org/cen/topstory/ 7912/7912notw5.html; Physics News Update: http:// www.aip.org/physnews/update/529-3.html; New Scientist: http://www.newscientist.com/tech/holographic.jsp; Optics.org: http://www.optics.org/article/news/07/5/13; Laser Focus World, p. 95, May 2001; Materials Research Society Bulleting 26, 277 (2001); Chem. Phys. Chem. 3, 333 (2002).

6. G. Ramos, A. Alvarez-Herrero, T. Belenguer, et al., Appl. Opt. 43, 4018 (2004).

7. L. Carretero, A. Murciano, S. Blaya, et al., Opt. Express 12, 1780 (2004).

8. J. Park and E. Kim, Key Eng. Mater. 1039, 277 (2005).

9. P. Cheben, F. del Monte, D. J. Worsfold, et al., Nature 408, 64 (2000).

10. F. del Monte, O. Martínez-Matos, J.A. Rodrigo, et al., Adv. Mater. 18, 2014 (2006).

11. N. Suzuki, Y. Tomita, and T. Kojima, Appl. Phys. Lett. 81, 4121 (2002).

12. C. Sanchez et al., Adv. Funct. Mater. 15, 1623 (2005).

13. J. A. Frantz, R. K. Kostuk, D. A. Waldman, Proc. SPIE 4296, 159 (2001). 